\title{
Modelo del proceso de enseñanza aprendizaje de los conceptos matemáticos en la formación del informático.
}

\author{
Model of the teaching learning process of mathematical concepts in the \\ training of computer engineers.
}

\author{
Roberto José Bueno Hernández. ${ }^{1}$ \& Walfredo González Hernández. ${ }^{2}$
}

\begin{abstract}
.
Introduction. Training competent computer engineers is a current need, so, the study of the teaching learning process of these professionals is essential to society's development. Aim. To elaborate a model of the teaching learning process of mathematical concepts and its definitions in the training of the computer engineer, to contribute to his professional development. Results and discussion. In this article, it is defined model, from the positions of the Theory of Subjectivity as a current trend of the historical cultural approach. This allows the authors to take sides about its structure and characterize its elements. In a second moment, the authors propose, from the assumed theory, a structure of the model to the teaching learning process of Mathematical concepts and its definitions in the training of computer engineers. Conclusion. The proposed model contributes to the comprehension of the educational reality in order to transform it.
\end{abstract}

Keywords: Model, Mathematical concepts, and Theory of Subjectivity

\section{Resumen.}

Introducción. Formar ingenieros informáticos competentes es una necesidad actual por lo que el estudio del proceso de enseñanza aprendizaje de estos profesionales es esencial parar el desarrollo de la sociedad. Objetivo. Elaborar un modelo del proceso de enseñanza

\footnotetext{
${ }^{1}$ Universidad de Matanzas, Facultad de Educación, Matanzas, Cuba. roberto.bueno@umcc.cu ORCID: http://orcid.org/0000-0002-5573-0049

${ }^{2}$ Universidad de Matanzas, Facultad de Ciencias Técnicas, Matanzas, Cuba. walfredo.gonzalez@umcc.cu ORCID: http://orcid.org/0000-0001-8974-3721
} 
aprendizaje de los conceptos matemáticos y sus definiciones en la formación del ingeniero informático para contribuir a su desarrollo profesional. Resultados y discusión. En este artículo se define modelo desde las posiciones de la Teoría de la Subjetividad, como vertiente actual del enfoque histórico cultural, lo que permite a los autores tomar partido acerca de su estructura y caracterizar los elementos de este. En otro momento se elabora desde la teoría asumida una propuesta de estructura del modelo para el proceso de enseñanza aprendizaje de los conceptos matemáticos y sus definiciones en la formación del ingeniero informático. Conclusión. Este modelo propuesto contribuye a la comprensión de la realidad educativa del informático en función de su transformación.

Palabras claves: Modelo, conceptos matemáticos y Teoría de la Subjetividad

\section{Introducción}

La realidad educativa es un fenómeno social que a lo largo de la historia y en diversas culturas se ha caracterizado por presentar ciertas regularidades epistemológicas que se difunden entre los profesores. También se conoce que el ser humano está condicionado para aprender a partir de las relaciones que se establecen durante su vida con el entorno en el cual se desarrolla. El hombre además, a lo largo de su historia ha salvaguardado su legado y conocimiento para las nuevas generaciones, lo cual ha constituido una de sus principales preocupaciones.

Este proceso de transmisión del conocimiento se le denomina enseñanza, y el proceso de apropiación y producción del conocimiento se le denomina aprendizaje. Investigaciones demuestran que las concepciones filosóficas, psicológicas, sociológicas, entre otras, determinan la representación social que pueden tener los sujetos acerca de estos procesos. El desarrollo del hombre ha generado ramas que del conocimiento que están vinculadas a procesos vitales como: la comunicación, las relaciones cuantitativas con el entorno, los estudios sobre la salud, el lenguaje, la enseñanza y el aprendizaje, entre otros.

El proceso de producción y desarrollo del conocimiento humano vinculado con los modelos educativos posibilita la emergencia de nuevos modelos educativos orientados a preservar el legado humano. Por lo que en constante perfeccionamiento de los modelos educativos es una garantía para desarrollar un adecuado proceso de enseñanza aprendizaje. La atención se centra entonces en los modelos de aprendizaje como proceso de continuidad de la humanidad.

Esta investigación se estableció a partir de identificar vacíos epistemológicos en la educación superior cubana y en especial en el proceso de enseñanza de los conceptos matemáticos y sus definiciones lo que permitieran comprender como desarrollar el proceso educativo. Una consecuencia de estos vacíos fue la falta de una teoría que permitiera comprender la congruencia y consistencia de las aportaciones de los grandes maestros con los métodos de enseñanza que aún hoy siguen vigentes en las aulas de clase. 
Para contribuir a la solución a los problemas planteados anteriormente esta investigación está dirigida a elaborar un modelo del proceso de enseñanza aprendizaje de los conceptos matemáticos y sus definiciones en la formación del ingeniero informático para contribuir a la comprensión de la realidad educativa en función de su transformación.

En el campo de las ciencias pedagógicas diversos autores Ruiz, (2002,) Guetmanova (1991), Sierra (2011), García (1997) Davidov (1980), han abordado los modelos y sus clasificaciones en función del problema que se han propuesto resolver. De manera general puede expresarse que su empleo en la pedagogía tiene gran importancia debido a que "se convierten en medio y método para lograr representaciones simples de fenómenos complejos como los que se presentan en la vida diaria y sobre todo en el área de las ciencias pedagógicas" (Valle de Lima, 2012, p. 135).

Las definiciones brindadas por los autores anteriores logran expresar el carácter teórico que tiene todo modelo. Cuestión tal que obtienen a partir de múltiples enfoques para aproximarse a determinar que es un modelo como objeto. En algunas se refieren al papel que juega la abstracción para la conformación del modelo. Sin embargo, se considera que este elemento debe ser abordado de otra manera debido a que se debe tener claro cuáles son los elementos o características de la realidad que no se pueden obviar para modelarla. Por otra parte, en esta investigación se parten de referentes teóricos que están basado en el análisis complejo de la realidad educativa, lo que implica el estudio de todos los factores que inciden en la realidad, así como las relaciones entre estos.

\section{Resultados y discusión.}

La autora Flores, M. (2019) define "modelo pedagógico" como:

Un esquema teórico-conceptual de la propuesta, construida a partir de la realidad compleja, para facilitar el conjunto de acciones que guían el proceso de aprendizaje y que por su excelencia en la aplicación y solución de problemas educativos se convierte en un prototipo de ser generalizado; asimismo formula técnicas y procedimientos para desarrollar la tarea de enseñar, también apoya en la comprensión y estudio de la evolución de los procesos educativos y su aplicación en el marco de diferentes posturas de pensamiento de las diversas culturas. (p. 145)

El modelo pedagógico es una guía (Ortiz, 2015) que se diferencia de otros términos similares que pueden llegar a confundirse: 'modelo educativo'; 'modelo didáctico' 'modelo de enseñanza'. Se podría decir que la diferencia entre los términos radica en los niveles de concreción y ejecución en la realidad educativa. Es conveniente aclarar que los tres constructos aludidos son holoárquicos (Wilber, 2011) he inclusivos entre sí; es decir, el concepto holoarquía hace referencia a los modelos educativo, pedagógico y didáctico como totalidades-parte con una jerarquía natural. Se diferencia de una jerarquía de dominio que es más artificial y conflictiva Cfr. (Wilber, 2011). El modelo educativo incluye y trasciende al modelo pedagógico y éste a su vez al modelo didáctico sin invadir sus niveles de concreción y ejecución. Existe un orden jerárquico en cuanto a niveles de 
actuación (macro, meso, micro), escala de concreción (nacional, escolar y áulico) y las implicaciones que se desprenden en cada uno de los conceptos estableciendo claramente sus límites e interrelaciones. (Flores, M. 2019). Existen investigaciones de diversos autores que están suscitadas por la incoherencia entre las definiciones de modelo y los referentes teóricos de la investigación, de ahí que sea necesario definir este concepto desde estos referentes.

Un modelo emerge a partir de la maduración de una concepción mediante la cual los procesos investigativos se desarrollan. La diferencia entre estos conceptos, más allá de su alcance está dado por el grado de sistematización que demanda el modelo sobre la concepción. En el modelo aparecen elementos particulares que permiten operar, desde lo cognitivo y axiológico con la realidad que se estudia, con el objetivo primordial de transformar el resultado de la actividad práctica hacia el estado deseado por el hombre en su conjunto.

Resulta esencial desde nuestra concepción y utilizando estos referentes teóricos, definir modelo como un sistema de configuraciones subjetivas que le permiten al hombre representarse la realidad mediante un sistema de símbolos que la describen, lo que propicia el conocimiento de las principales normas (principios, leyes y categorías) que la rigen, lo cual sustenta el emprendimiento de acciones en función de su transformación acorde a las aspiraciones humanas.

En lo referido al sistema de símbolos mediante el cual el hombre se representa la realidad, estos incluyen palabras, esquemas, signos (en particular matemáticos), gráficos y otras expresiones externas; mientras que a lo interno del sujeto estos actúan como razonamientos, ideas, conceptos, leyes, entre otras formas del pensamiento abstracto.

Como se abordó anteriormente todos los modelos constituyen en sí mismos un resultado teórico. En el marco de esta investigación se considera que los modelos teóricos alcanzan la condición de modelos metodológicos cuando integran en sí acciones para orientar al profesor en la conducción del proceso de enseñanza aprendizaje.

A partir de todo lo anterior se coincide con Valle de Lima (2012) en que todo modelo surge por un fin y tiene objetivos que conducen su elaboración, principios que constituyen puntos de partida para la construcción teórica del modelo que pueden estar acompañados por leyes y un sistema categorial donde se caracterice el objeto de la investigación. Este debe contener una estrategia metodología, entre otras, que indique como debe implementarse en la práctica educativa. Así como determinadas formas de evaluar la validez del modelo y su contribución a la teoría y la práctica.

Para construir un modelo de aprendizaje es necesario que se constituyan once elementos básicos estructurantes que permitirán establecer niveles de comprensión teóricometodológica y conforme a las características del modelo: congruencia, consistencia y coherencia; además que promueven una caracterización y comprensión de la realidad educativa que se intenta representar; a partir de establecer una relación entre los siguientes elementos: 1) un contexto socio-histórico de surgimiento del modelo; 2) una definición 
conceptual, 3) su fundamento filosófico-epistemológico; 4) el fundamento psicológico, 5) los fines de la educación, 6) contenidos del curriculum, 7) noción de aprendizaje, 8) rol del estudiante, 9) rol del maestro, 10) estrategias de enseñanza y 11) dispositivos de evaluación. Se considera que estos elementos estructurantes no son exhaustivos, pero si representan un panorama global del modelo pedagógico (Flores, M. 2019) y en este caso del modelo de aprendizaje que es lo que se desea estudiar.

De los referentes analizados se pudiera resumir los siguientes aspectos necesarios para elaborar un modelo para los procesos de la educación como es el aprendizaje:

- Forma de obtención de los elementos del modelo.

- Sistema de elementos básicos que componen la estructura.

- Interrelación con otros modelos que conforman el ecosistema donde se incluye.

Los aspectos obtenidos guiarán la conformación del modelo del proceso de enseñanza aprendizaje de los conceptos matemáticos y sus definiciones en la formación del ingeniero informático que será desarrollado a continuación.

\section{Modelo del PEA de los conceptos matemáticos y sus definiciones.}

El objetivo del modelo de aprendizaje que se propone es contribuir al perfeccionamiento del proceso de enseñanza aprendizaje de los conceptos matemáticos y sus definiciones en la formación del ingeniero informático debido a la importancia que estos tienen para su formación profesional.

Es necesario develar las interrelaciones entre los componentes del modelo, así como las particularidades de cada uno de ellos, por lo que asumiremos una representación esquemática que se encuentra en la figura 1 donde se presenta la estructura del Modelo de Aprendizaje Desarrollador de la Informática, el cual se considera que se pudiera extrapolar al proceso de enseñanza aprendizaje de los conceptos matemáticos y sus definiciones:

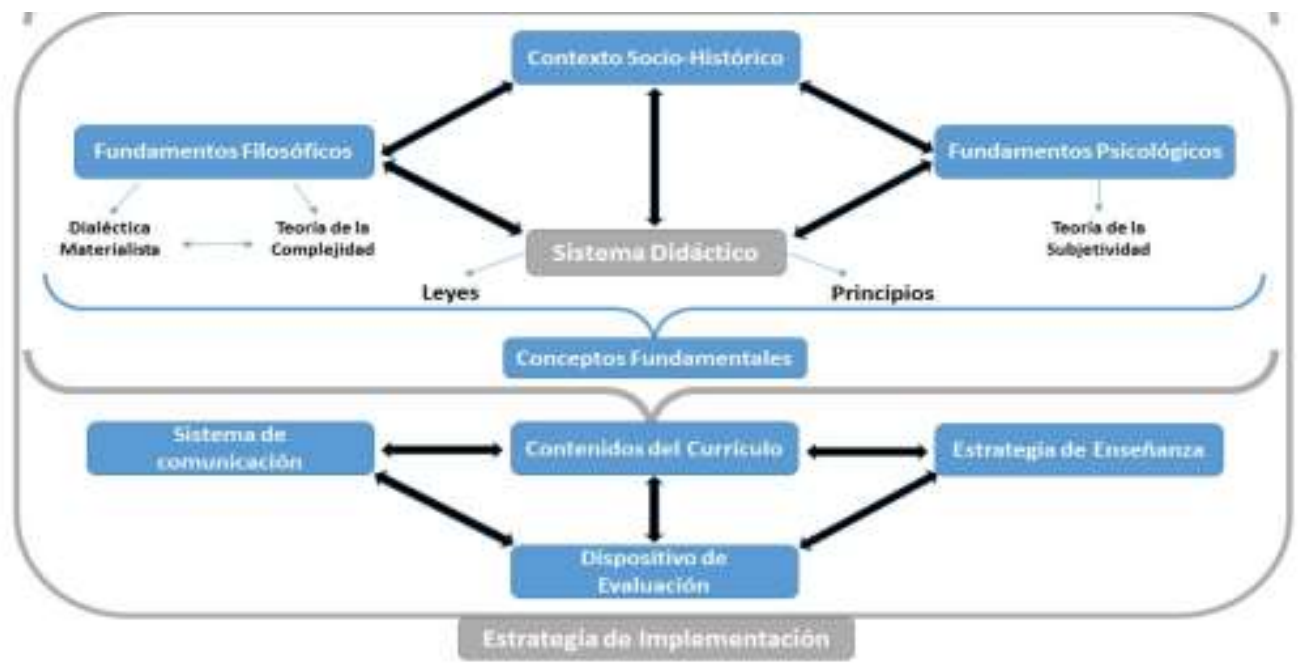

Figura 1: Estructura del Modelo de Aprendizaje Desarrollador de la Informática. Fuente: Elaboración propia 
El presente modelo de aprendizaje presenta varias características que se argumentan a continuación:

- Abierto: Este se concibe adaptable a cualquier escenario educativo de la Educación Superior en el que se pretenda enseñar Matemática a un ingeniero informático

- Flexible: El modelo está concebido para tener en cuenta las características individuales de los sujetos que intervienen en la realidad educativa

- Dinámico: La relación entre sus componentes no es estática, sino se cambia a la par de las condiciones externas.

- Configuracional: Su estructura se modifica según los roles y las relaciones que se establecen entre sus componentes en constante cambio.

El modelo se puede clasificar como un modelo híbrido pues la vía inductiva proviene de las investigaciones empíricas en la educación superior que critican los modelos educativos imperantes y proveen de inferencias interesantes sobre el deber ser del proceso. Al mismo tiempo, el análisis de diferentes fuentes bibliográficas declaradas sobre la Teoría de la Subjetividad permite inferir las características esenciales del modelo.

\section{Contexto socio-histórico de surgimiento del modelo}

El modelo surge en un contexto socio histórico de auge de las tecnologías informáticas y de las comunicaciones (TICs) que ha impactado en la sociedad a niveles muy altos. En la actualidad muchas de las actividades humanas tienen en las TICs un sustento que incrementa la eficiencia y la eficacia. Esta es una de las muchas razones por las que el desarrollo de las tecnologías se considera estratégico para el desarrollo de los países, por lo que la preparación de profesionales en estas tecnologías se torna impostergable.

En este contexto existen dificultades en el proceso de enseñanza aprendizaje de forma general, pero en particular en la matemática que a su vez es fundamental para el desarrollo de la Informática. Una de estas dificultades relacionadas con la enseñanza de la Matemática está en la poca vinculación de los contenidos que se imparten con la práctica, y en este caso en particular, a la informática lo que conlleva a ser un proceso formativo que se orienta al futuro.

Otra de las problemáticas del contexto socio histórico está en el cuestionamiento de las universidades como organizaciones formadoras de profesionales.

La tercera problemática plantea serios desafíos a las universidades, pues cuestiona su función social fundamental, los procesos que tienen lugar en ella, así como sus resultados. La integración de los procesos sustantivos universitarios y productivos puede llevar a que la formación profesional sea conducida por profesionales de estas organizaciones no educativas sin una formación docente. Al mismo tiempo, provee al estudiante de un entorno más cercano a su futuro puesto laboral, lo que puede llevar a reformular o afianzar su proyecto de vida profesional. Esta integración provee al estudiante de nuevos espacios de aprendizaje que plantea a la universidad dinámicas diferentes donde los errores 
estudiantiles deben ser mitigados porque tienen un impacto en los procesos empresariales. (Bueno, Naveira, \& González, 2020)

Desde el punto de vista filosófico el modelo se sustenta en la dialéctica materialista en diálogo con la Teoría de la Complejidad y la Teoría General de Sistemas. Esta posición filosófica asumida permite estructurar un sistema adecuado de principios teóricos y metodológicos. Estos principios posibilitan comprendes la realidad educativa como un proceso multivariados, concatenado y multifactoriales, integrados en relaciones que determinan la configuración de los sistemas que se estudian.

Sociológicamente este modelo está fundamentado en la educación condicionada desde lo histórico-social, dirigida a la formación y desarrollo del sujeto a través de la transmisión y apropiación de la herencia cultural de la humanidad mediante el sistema de influencias de los variados niveles de socialización. Este modelo desde lo Sociológico está en correspondencia con los fines de la educación y del proyecto social socialista cubano y con la política de formación del profesional revolucionario. De forma más amplia está en correspondencia con la formación de un profesional portador de una cultura general integral. En la enseñanza de la Matemática se debe dotar de contenidos y conceptos matemáticos al sujeto para que este alcance objetivos que son necesarios a nivel social, como por ejemplo: la concepción científica del mundo, el pensamiento lógico y la resolución de problemas. La matemática como herramienta esencial para la comprensión y transformación de la realidad, la precisión del lenguaje y el gusto por la exactitud, por solo mencionar algunas funciones, es una concepción que depende en gran medida de nivel de aplicación de los conceptos matemáticos.

El fundamento psicológico del modelo es el enfoque histórico cultural de L. S. Vygotsky (Vygotsky, 1995), en su vertiente de la Teoría de la Subjetividad de Fernando González Rey (González Rey \& Mitjáns Martínez, 2019). Las categorías sentido subjetivo, configuración subjetiva y perezhivanie constituyen los pilares fundamentales en la investigación, ya que permite explicar cómo los procesos simbólicos implicados en el contenido de enseñanza que debe aprender el estudiante, se integran a procesos emocionales, formando relaciones, de manera que se desarrolle un proceso recursivo. Al asumir las configuraciones subjetivas como sistemas que se integran entre sí de modo recursivo se puede entender cómo transcurre el aprendizaje de los sujetos basado en este paradigma.

Dentro de los fundamentos pedagógicos se reconocen el sistema de relaciones entre los componentes del proceso mencionados con anterioridad (sociedad, familia, escuela, profesor, grupo y estudiante), cuyo carácter configuracional (González-Hernández, 2016) permite dinamizar e integrar los sentidos subjetivos, así como las configuraciones subjetivas que se generan en el estudiante durante el proceso educativo. El profesor como sujeto activo en la conducción del proceso docente educativo es un elemento que se caracteriza por las funciones que debe cumplir, (metodológica, investigativa y orientadora) (Labarrere Reyes \& Valdivia Pairol, 1988). 
Desde el aspecto didáctico se comprende el proceso de enseñanza aprendizaje de los conceptos matemáticos y sus definiciones como: sistema complejo, cuya configuración posibilita la emergencia de nuevas propiedades que distinguen cada "clase", como momento esencial para que se desarrolle la relación entre los componentes que intervienen en el proceso. Este proceso se debe desarrollar de forma que emerjan en el estudiante nuevos sentidos subjetivos asociados al aprendizaje, cuya integración en configuraciones subjetivas favorables a este último resulta esencial para que los estudiantes se apropien del contenido y venzan los objetivos del currículo.

Las leyes del proceso asumidas son:

"La relación configuracional entre la sociedad y la educación" (González-Hernández, 2016, p. 93): Desde esta ley se puede explicar por qué los procesos educativos no siempre cumplen con el encargo social y esta relación no es determinística.

"Sistema complejo compuesto por varios subsistemas con interacciones configuracionales" (González-Hernández, 2016, p. 100): las interacciones que se dan entre los encargados de enseñar y los encargados de aprender así como el resto de los componentes y categorías de la didáctica se configuran en el acto didáctico. Para que estas interacciones ocurran debe determinarse diversos elementos que van desde la planeación escolar hasta el contenido de enseñanza perneados por las concepciones que tienen los sujetos que en él intervienen.

Los principios se sintetizan en los siguientes (González-Hernández, 2021):

El principio del carácter subjetivo del aprendizaje: este principio propone asumir que el aprendizaje es un proceso productor de subjetividades tanto para los que se encargan de enseñar como para los que aprenden. Por tanto, los diseños curriculares, así como los espacios de aprendizaje deben tener momentos para generar producciones subjetivas tanto individuales como sociales en los cuales la comunidad y la familia tienen un espacio importante.

El principio del carácter configuracional y complejo de educación: parte de la idea que el aprendizaje es un proceso multifactorial y multicultural en el cada uno de los factores no siempre tiene el mismo peso. Al mismo tiempo, cada uno de ellos se integran de manera única e irrepetible en cada proceso educativo, ya sea curricular, de gestión, clase o el mismo aprendizaje. Ello explica que la unicidad de cada proceso a nivel de país, organización educativa, y hasta los espacios de aprendizaje donde ellos se concretan.

El principio de la integración entre la sociedad y la universidad: La universidad es parte integrante de una sociedad con la cual establece interacciones configuracionales. La formación de los profesionales puede transcurrir en los espacios de las organizaciones comunitarias donde se integran los sentidos subjetivos asociados a su profesión en configuraciones subjetivas sociales con aquellos profesionales que se desempeñan en ellas. 
Por otra parte, la ciencia Matemática provee herramientas que favorecerán al estudiante en su futuro desempeño profesional. Esta ciencia contribuye con procesos como comprender el problema, abstraer, modelar y construir (Anhalt, Staats, Cortez, y Civil, 2018) además de evaluar los proyectos previos al desarrollo de un producto informático. También, muchos de los sectores productivos exigen a los informáticos una formación integral para su ejercicio profesional. Dentro de esta formación se debe lograr un desarrollo del pensamiento lógico, la resolución de problemas y la capacidad de abstracción, lo que se obtiene con el dominio de los contenidos de su currículo, pero en especial de la matemática.

Una adecuada formación matemática es necesaria durante el proceso de formación de un ingeniero informático, pero para lograrlo se necesita una fuerte formación de los conceptos propios de esta ciencia exacta. Esta permite representar la relación entre la matemática y la realidad objetiva, por lo que es necesario reflexionar sobre como lograr que los estudiantes comprendan que los conceptos, al igual que las formas de trabajo matemático, surgen en la práctica cotidiana del hombre ante la necesidad de transformar la realidad. Se considera entonces que si la formación de los conceptos matemáticos es imprescindible para la matemática a la misma vez que esta última es básica en la formación de un ingeniero informático y, por las características de la informatizada sociedad actual, entonces no es posible sostener ni desarrollar la sociedad sin tener un conocimiento de los conceptos matemáticos.

\section{Conceptos fundamentales del modelo:}

Proceso de enseñanza aprendizaje: como configuración subjetiva social en la que intervienen quien enseña y quien aprende, que ocurre en el sistema de espacios de aprendizaje o en uno de ellos en tensión con determinados objetivos, de acuerdo a la situación social del desarrollo de todos los involucrados.

Conceptos matemáticos: constituyen una producción subjetiva del sujeto en función de la integración de los sentidos subjetivos en torno a la abstracción que lleva a la formación del concepto. El concepto para formarse no siempre tiene que visualizarse el objeto, esto posibilitaría organizar un sistema de acciones para comprenderlo, que puede transformarse durante la interacción.

Proyecto de vida:

... formación psicológica de la personalidad integradora de sus direcciones vitales principales implica, de una parte, las relaciones de todas las actividades sociales de la persona (trabajo, profesión, familia, tiempo libre, actividad cultural, socio-política, relaciones interpersonales de amistad y amorosas, organizacionales, etc.); de otra, es la expresión del funcionamiento de diferentes mecanismos y formaciones psicológicas que integran todo el campo de la experiencia personal (D’Angelo, 2015, p. 4). 
Sentido subjetivo como la “... unidad de los procesos simbólicos y emocionales donde la emergencia de uno de ellos evoca al otro sin convertirse en su causa, formando verdaderas cadenas con formas muy diversas de expresión según el contexto en que la persona está implicada” (González-Rey, 2011, p. 3)

Situación Social del Desarrollo como:

...aquella relación peculiar, única, especial e irrepetible entre el sujeto y su entorno que va a determinar las líneas de desarrollo, la forma y trayectoria que permiten al individuo adquirir nuevas propiedades de la personalidad, considerando a la realidad social como la primera fuente de desarrollo, la posibilidad de que lo social se transforme en individual (González-Rey, 2010, p. 18)

Reestructuración de los componentes personales del proceso (González Hernández, 2021): vinculando los dos últimos principios, los procesos formativos universitarios pueden ser conducidos por profesionales de las organizaciones comunitarias, los que no siempre tienen formación pedagógica para conducir procesos de formación profesional. Al mismo tiempo, otros actores del proceso educativo que no están formados como profesores, de la rema específica, interactúan con los estudiantes y les enseñan. En el caso de la matemática en ocasiones los profesores no son matemáticos de formación pedagógica, por lo que el tratamiento de los conceptos y sus definiciones no se realiza de forma profunda siendo esto fundamental. Por tanto, se asumen tres componentes fundamentales: el grupo, los que aprenden y los que enseñan pues los estudiantes pueden enseñar a los profesores y viceversa.

El concepto de espacio de aprendizaje: El concepto amplía el concepto de aula como el espacio por excelencia para el aprendizaje cuando define que un espacio de aprendizaje es "... un espacio de producción subjetiva a través del diálogo entre los componentes del proceso pedagógico (en su sentido más general) en el cual transcurre el aprendizaje" (González Hernández, 2021). De la definición se desprende que los espacios de aprendizaje pueden ser una combinación de espacios virtuales y físicos en los cuales el sujeto o los sujetos que lo construyen se implican para aprender.

El concepto de problema: La categoría problema es la piedra angular de la enseñanza problémica y generalmente está asociado a una situación contradictoria para un individuo; sin embargo, estas definiciones no explican el por qué son contradictorias. Para Bueno Hernández, González Hernández, and Naveira Carreño (2020) un problema es “... producción subjetiva que sucede al interactuar con una situación de la cual emergen sentidos subjetivos que provocan tensiones con las configuraciones subjetivas constituidas en el individuo"(p. 116). Esta definición tiene en cuenta que una situación puede ser ambivalente para un estudiante si no hay producción simbólica - emocional que la lleve a ser contradictoria para él. Esta concepción asume que una situación problémica expuesta por un profesor puede no ser problémica para todos los estudiantes.

\section{Los contenidos del currículo}


Los contenidos curriculares de la Matemática se agrupan en varias disciplinas científicas que les permiten informatizar con éxito las organizaciones. Una breve descripción de cada una de ellas permitirá caracterizar las asignaturas:

- $\quad$ Matemática Superior juega un papel primordial ya que esta contribuye a que los futuros egresados adquieran una concepción científica del mundo, al desarrollo del pensamiento lógico y algorítmico y aporta los fundamentos básicos de contenidos propios del ejercicio de la profesión. Esta disciplina aporta al estudiante los conocimientos necesarios para su formación académica y las herramientas de trabajo que permite identificar, interpretar y analizar modelos matemáticos en procesos técnicos, económicos, productivos y científicos vinculados a su profesión. Esta disciplina permite asumir una concepción científica del mundo al interpretar los conceptos del Cálculo Diferencial e Integral, el Algebra Lineal, la Geometría Analítica, las Series, las Ecuaciones Diferenciales y la Matemática Numérica, como resultados de la Ciencia Matemática.

- Inteligencia Computacional (IC) esta disciplina tiene sus orígenes en las disciplinas Matemática Aplicada e Inteligencia Artificial. Ejemplos de estos contenidos son la lógica matemática, el cálculo proposicional, la minimización de funciones lógicas y de círculos lógicos. También la modelación matemática y la programación lineal era uno de los temas de investigación que más se trabaja en el centro de estudios de ingeniería y sistemas (CEIS). Esta disciplina se enfoca en el modelado y solución de problemas complejos usando métodos avanzados usando sistemas formales con base en la matemática y la lógica.

- Ingeniería y Gestión de Software (IGS) Según el plan de estudio E en esta disciplina los estudiantes necesitan de un pensamiento algorítmico, de la modelación, de técnicas de estimación, desarrollar en lenguaje de programación, resolver problemas entre otros contenidos donde la ciencia Matemática es fundamentar para un correcto desarrollo del proceso.

- Infraestructura de Sistemas Informáticos (ISI) en ella la Física es una de las asignaturas que la integran por lo que la matemática es la base de ese sistema de conocimientos. También en esta disciplina el sistema de numeración binario y hexadecimal, así como la modelación y la resolución de problemas son conocimientos fundamentales para la adecuada formación del ingeniero informático.

- Práctica Profesional esta disciplina está concebida como una disciplina integradora de la carreta. Esto se aprecia ya que en esta tiene que aplicar los conocimientos impartidos en las demás asignaturas de otras disciplinas.

- $\quad$ Estas disciplinas, mencionadas con anterioridad, entre otras tienen una gran carga de contenidos matemáticos y en especial de conceptos matemáticos. Muchas de estas intervienen en mayor o menor grado durante los procesos de informatización de las organizaciones en dependencia del tipo de proyecto, pero en cualquiera de los casos necesitan una sólida formación de conceptos 
matemáticos. Si se trata de un proceso de desarrollo de software de gestión, generalmente intervienen todas excepto la cuarta; pero si se trata de modelar alguna forma de actuación humana es la cuarta la preponderante. De ahí que los estudiantes deben estar preparados con los elementos necesarios para desarrollar estos procesos.

Sistema de comunicación: la reafirmación de los que deben aprender como sujetos en los espacios de aprendizaje debe estar sustentada en el establecimiento de interacciones basadas en el diálogo donde la aceptación del otro juegue un papel fundamental. De esta manera, los conceptos matemáticos como producciones subjetivas de todos los sujetos que componen el espacio, van integrándose por lo que emergen sentidos subjetivos de pertenencia y comienza su conversión en lugar (Esteban-Guitart \& Llopart, 2016) y así se van conformando conceptos, prácticas, normas que serán institucionalizadas. También el diálogo va aportando el elemento de unión de los componentes personales y van emergiendo sentidos subjetivos asociados a los conceptos matemáticos que favorezcan la constitución de una configuración subjetiva social en forma de comunidad de aprendizaje.

\section{Estrategias de enseñanza:}

Metodológicamente, existen requerimientos que dictan condiciones fundamentales que el profesor debe garantizar para la conducción del proceso de enseñanza aprendizaje.

Emergencia de procesos emocionales asociados a la matemática: el profesor debe valerse de ciertos recursos que motiven al estudiante, de manera que pueda influir en sus gustos, intereses, opiniones y concepciones sobre la Matemática. Algunos de los recursos que se recomiendan son: las aplicaciones de la disciplina Matemática Superior a la Ingeniería Informática, la historia de la Matemática, las curiosidades matemáticas, la etnomatemática, entre otros. Sobre todo desde el diagnóstico de estos sentidos subjetivos asumiendo una postura de diálogo constante con los estudiantes que les permita explorarlos y el apoyo necesario para que se transformen en emociones positivas.

Cuando aplicamos los recursos mencionados anteriormente en el desarrollo de una clase o de un sistema de clases se contribuye a que se integren los procesos emocionales y simbólicos de la Matemática Superior, lo que permite la emergencia de sentidos subjetivos asociados al contenido de enseñanza de la disciplina. Estos a su vez, se deben integrar en configuraciones subjetivas que propicien una variación de la perezhivanie del estudiante, de manera que esta esté en armonía con los objetivos propuestos para el aprendizaje de los conceptos matemáticos y sus definiciones. Estos procesos deben tener un carácter sistemático durante el desarrollo de la disciplina o asignatura.

Evidenciar las relaciones entre los conceptos matemáticos y sus definiciones, y la arista profesional del proyecto de vida del estudiante: es necesario que el profesor tenga una sólida preparación y que exista un trabajo integrado del colectivo de año para lograr estos fines. Esto favorece el requerimiento anterior, dado que existen sentidos subjetivos asociados a su proyecto de vida, los que se deben integrarse a otros procesos emocionales y simbólicos asociados a la Matemática Superior. 
Asegurar las condiciones previas en cada estudiante: El profesor debe identificar qué estudiantes no se han apropiado del concepto matemático y su definición como parte esencial del contenido de enseñanza de la asignatura, para lo cual debe preparar actividades que garanticen las bases para un desarrollo favorable de la disciplina.

Orientar a los estudiantes en cada actividad: Los estudiantes deben entender el objetivo de cada acción que realizan, para lograr su implicación en el desarrollo de esta. De igual modo deben conocer las metas a alcanzar en la disciplina a corto, mediano y largo plazo, así como la importancia de su consecución para su formación como ingeniero informático.

Garantizar el protagonismo de los estudiantes durante su aprendizaje: Es necesaria la implicación de los estudiantes en la elaboración de un nuevo concepto. Esto se alcanza a través de un proceso comunicativo que propicie su participación, donde se deben emplear métodos productivos de enseñanza aprendizaje. La vía inductiva resulta esencial para ello, ya que los procesos de razonamiento respecto a las acciones realizadas, para obtener regularidades, contribuyen al desarrollo del pensamiento lógico de los estudiantes y los involucran emocionalmente con el contenido que debe aprender, de manera tal que estos se tornen sujetos de su aprendizaje.

Evaluar el logro de los objetivos: El profesor debe, en todo momento, hacer una valoración sobre cómo los estudiantes consiguen los objetivos que se proponen. Este proceso debe tener un carácter sistemático que no necesariamente se expresa a través de una calificación. Los instrumentos de evaluación a utilizar se deben elaborar cuidadosamente, de forma que identifiquen en qué nivel se cumplieron estos objetivos propuestos. Debe ser, además, un proceso en el que el estudiante se involucre, de modo que se sienta estimulado para demostrar los conocimientos y habilidades que ha adquirido. Es el profesor quien debe generar esta atmósfera al emplear formas creativas de evaluación que favorezcan el correcto desarrollo del estudiante, como pueden ser a través de proyectos, portafolios o autoevaluaciones entre otras.

Estos requerimientos metodológicos muestran las condiciones que el profesor debe garantizar, así como acciones metodológicas que aseguran la funcionalidad del modelo de aprendizaje en la práctica, de modo que se logre, en los estudiantes, el aprendizaje de los conceptos matemáticos y sus definiciones en la formación del ingeniero informático.

\section{Dispositivos de evaluación:}

La evaluación del proceso de enseñanza aprendizaje de los conceptos matemáticos y sus definiciones cumplirá con todas las funciones didácticas. También se debe prestar esencial atención a las configuraciones subjetivas para aprender, las cuales se diagnosticarán sistemáticamente a través de instrumentos de investigación abiertos. Es importante tener presente todos los símbolos y emociones que van emergiendo durante el propio proceso por lo que una buena comunicación entre el que aprende y el que enseña tiene que ser primordial. Ello le permite al estudiante reflexionar sobre lo que ha hecho y lo que debe hacer utilizando para ello registros accesibles cuando los necesite, 
potenciando la autoevaluación y la coevaluación. Ello potencia el desarrollo del estudiante llevándolo a la emergencia de sentidos subjetivos integrándose en configuraciones subjetivas en un proceso recursivo que va formando al profesional de la informática.

En este modelo se privilegia la evaluación continua a partir de los instrumentos periódicos que se hacen en el desarrollo del proceso donde el estudiante se implica activamente. Son los colectivos de año y de disciplina los encargados de evaluar el desarrollo de cada estudiante y sus posibilidades reales para dar solución a las problemáticas asignadas relacionadas con su profesión con el uso de los conceptos matemáticos. De ahí que el clima de respeto y aceptación del error como parte del proceso de aprendizaje es esencial para la emergencia de sentidos subjetivos favorables para aprender. En todo momento el estudiante posee claridad de los resultados que obtiene, los que se esperan de él y hacia dónde debe dirigir sus esfuerzos que fundamentalmente está orientado al objetivo que la organización espera de él.

\section{Conclusiones.}

- La elaboración del modelo del proceso de enseñanza aprendizaje de los conceptos matemáticos y sus definiciones en la formación de ingeniero informático permitió, en primera instancia, proponer una solución al problema planteado. Este se concibió a partir de una definición aportada por el autor de la investigación en la que se detalla el alcance de todo modelo.

- El empleo del método "modelación" le permitió al autor formular los componentes del modelo así como sus elementos. Se considera que en cada componente se presentan y explican los elementos que conforman al modelo y que lo dotan de funcionalidad y objetividad

- Este resultado debe ser implementado para solventar en la práctica el problema generador del proceso investigativo que se acomete. Para lo cual se propone una estrategia de implementación.

\section{Referencias bibliográficas.}

Anhalt, C. O., Staats, S., Cortez, R., \& Civil, M. (2018). Mathematical modeling and culturally relevant pedagogy. In Cognition, metacognition, and culture in STEM education (pp. 307-330). Springer, Cham.

Bueno Hernández, R. J., González Hernández, W., \& Naveira Carreño, W. J. (2020). Análisis crítico acerca de la resolución de problemas desde la teoría de la subjetividad. Alternativas cubanas de psicología, 8(23), 112-125.

Bueno Hernández, R., Naveira Carreño, W., \& González Hernández, W. (2020) Los conceptos matematicos y sus definiciones para la formacion de los ingenieros informaticos para la sociedad. Universidad Y Sociedad, 12(4), 147-155. Recuperado a partir de http://rus.ucf.edu.cu/index.php/rus/article/view/1625 
D’Angelo, O. (2015). Formación para el desarrollo de proyectos de vida reflexivos y creativos en los campos social y profesional. Revista Crecemos Internacional, $5(2), 1-25$.

Davidov, V. V. (1980). Tipos de generalización en la enseñanza, . La Habana: Ed. Pueblo y Educación.

Esteban-Guitart, M., \& Llopart, M. (2016). La inclusión educativa a través de la aproximación de los fondos de conocimiento e identidad. Revista nacional $e$ internacional de Educación Inclusiva, 9(3), 145-157.

Flores, M. (2019). Cuatro formas de entender la Educación: modelos pedagógicos, conceptualización ordenamiento y construcción teórica. Educación y Humanismo, 21(36), 137-159.DOI: http://dx10.17081/eduhum.21.36.3147

García, L. (1997). Sistemas, modelos y teorías. material mimeografiado, ICCP.

González-Hernández, W. (2016). Las leyes de la didáctica y la realidad escolarizada. ¿Necesidad de cambio? Revista Latinoamericana de Estudios Educativos, XLVI(3), 85-110.

González-Hernández, W. (2021). Didactic principles: A proposal from the theory of subjectivity. Culture \& psychology. doi:10.1177/1354067x20984355

González Hernández, W. (2021). Los espacios de aprendizaje y las formas de organización de la enseñanza: una caracterización desde la subjetividad. Revista de Estudios y Experiencias en Educación, 20(42), 313-328. doi:10.21703/rexe.20212042gonzalez18

González Rey, F., \& Mitjáns Martínez, A. (2019). The Constructive-Interpretative Methodological Approach: Orienting Research and Practice on the Basis of Subjectivity. In F. G. Rey, A. M. Martínez, \& D. M. Goulart (Eds.), Subjectivity within Cultural-Historical. Approach Theory, Methodology and Research (Vol. 5, pp. 37-60). Singapure: Springer Nature Singapore.

González Rey, F. (2011). Sentidos subjetivos, lenguaje y sujeto: avanzando en una perspectiva postracionalista en psicoterapia. Rivista di psichiatria, 46(5 - 6), 310 $-314$.

González Rey, F. (2010). Las categorías de sentido, sentido personal y sentido subjetivo en una perspectiva histórico-cultural: un camino hacia una nueva definición de subjetividad. Universitas Psychologica, 9(1), 241-253.

Guetmanova, A. y. o. (Ed.) (1991). Moscú: Ed. Progreso.

Labarrere Reyes, G., \& Valdivia Pairol, G. J. (1988). Pedagogía. La Habana: Editorial Pueblo y Educación. 
Ortiz, A. (2015) Los modelos pedagógicos desde una dimensión psicológica-espiritual. Revista Científica General José María Córdova 13(15), 183-194.

Ruiz, A. (2002). Metodología de la investigación. LaHabana: Ed. Pueblo y Educación.

Sierra, V. (2011). Metodología de la Investigación Científica. La Habana: Felix Varela.

Valle de Lima, A. (2012). La investigación pedagógica. Otra mirada. La Habana: Editorial Pueblo y Educación.

Vygotsky, L. S. (1995). Pensamiento y Lenguaje: Teoría del desarrollo cultural de las funciones psíquicas. Argentina: Ediciones Fausto.

Wilber, K. (2011) Breve historia de todas las cosas. 5a . Ed. Barcelona: Kairós.

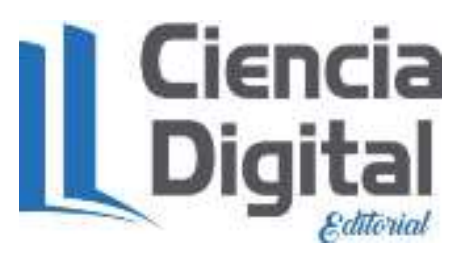




\section{PARA CITAR EL ARTÍCULO INDEXADO.}

Bueno Hernández, R. J., \& González Hernández, W. (2021). Modelo del proceso de enseñanza aprendizaje de los conceptos matemáticos en la formación del informático . Explorador Digital, 5(4), 27-43. https://doi.org/10.33262/exploradordigital.v5i4.1879

\section{Ciencia \\ Ligital \\ Edtorial}

El artículo que se publica es de exclusiva responsabilidad de los autores y no necesariamente reflejan el pensamiento de la Revista Explorador Digital.

El artículo queda en propiedad de la revista y, por tanto, su publicación parcial y/o total en otro medio tiene que ser autorizado por el director de la Revista Explorador Digital.
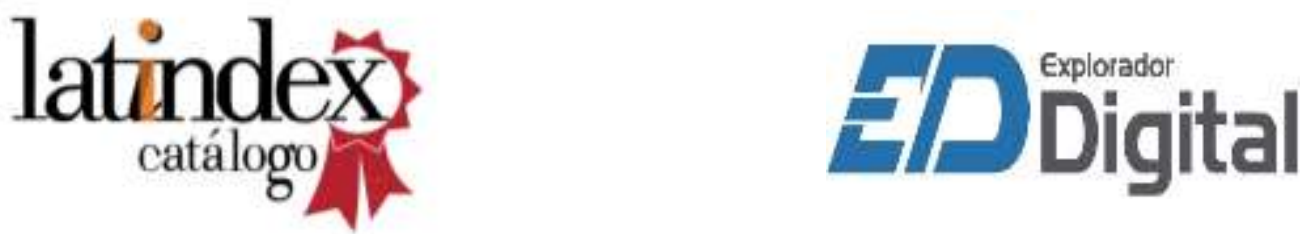